\section{Capacity of dental equipment to interfere with cardiac implantable electrical devices}

\author{
Lahor-Soler E, Miranda-Rius J, Brunet-Llobet L, Sabaté de la Cruz X. Capacity of \\ dental equipment to interfere with cardiac implantable electrical devices. \\ Eur J Oral Sci 2015; 00: 000-000. (C) 2015 Eur J Oral Sci
}

\section{Eduard Lahor-Soler ${ }^{1}$, Jaume Miranda-Rius ${ }^{1}$, Lluís Brunet- Llobet $^{2}$, Xavier Sabaté de la Cruz $^{3}$}

${ }^{1}$ Departament d'Odontostomatologia, Facultat d'Odontologia, Universitat de Barcelona, Barcelona; ${ }^{2}$ Servei d'Odontologia, Hospital Sant Joan de Déu, Universitat de Barcelona, Barcelona; ${ }^{3}$ Servei de Cardiologia, Unitat d'Arítmies, Hospital de Bellvitge, Facultat de Medicina, Universitat de Barcelona, Barcelona, Spain
Patients with cardiac implantable electrical devices should take precautions when exposed to electromagnetic fields. Possible interference as a result of proximity to electromagnets or electricity flow from electronic tools employed in clinical odontology remains controversial. The objective of this study was to examine in vitro the capacity of dental equipment to provoke electromagnetic interference in pacemakers and implantable cardioverter defibrillators. Six electronic dental instruments were tested on three implantable cardioverter defibrillators and three pacemakers from different manufacturers. A simulator model, submerged in physiological saline, with elements that reproduced life-size anatomic structures was used. The instruments were analyzed at differing distances and for different time periods of application. The dental instruments studied displayed significant differences in their capacity to trigger electromagnetic interference. Significant differences in the quantity of registered interference were observed with respect to the variables manufacturer, type of cardiac implant, and application distance but not with the variable time of application. The electronic dental equipment tested at a clinical application distance $(20 \mathrm{~cm})$ provoked only slight interference in the pacemakers and implantable cardioverter defibrillators employed, irrespective of manufacturer.
Jaume Miranda-Rius, Departament d'Odontostomatologia, Facultat d'Odontologia, Universitat de Barcelona, Feixa Llarga, s/n, 08907 L'Hospitalet de Llobregat, Barcelona, Spain

E-mail: jmiranda-rius@ub.edu

Key words: cardiac implantable electrical devices; dental surgical equipment; electromagnetic interference; implantable cardioverter defibrillator; pacemaker

Accepted for publication March 2015
Cardiac implantable electrical devices (CIEDs), which include pacemakers (PMs) and implantable cardioverter defibrillators (ICDs), are electronic appliances that are capable of analyzing the heart's rhythm and regulating cardiac arrhythmia through an electrical stimulus. Cardiac implantable electrical devices are typically placed subcutaneously, through a surgical procedure, in the left infraclavicular region and are connected by flexible electrode leads via the subclavian vein.

Pacemakers monitor the electrical activity of the heart and provide stimulation and heart conduction through electrical pulses. Implantable cardioverter defibrillators permanently control heart rate, and only in the case of specific arrhythmias do they emit electrical discharges.

In spite of the fact that present-day CIEDs possess protective mechanisms that recognize and filter most interference, some electromagnetic currents could temporarily affect their function (1). Electromagnetic interference takes place when the electromagnetic field of an electromagnet, or the flow of electricity generated by an instrument, interferes with the normal working of a nearby appliance. Electromagnetic signals, if they are affecting CIEDs, can produce electrical noise or even simulate electrical activity of the heart (1-5).
Dental practice frequently involves the use of sophisticated electronic and electromagnetic equipment within the oral cavity. The proximity of the lower third of the face with the infraclavicular region, where CIEDs are usually implanted, could augment the risk of interference in their function (5).

In odontology, an ultrasonic dental scaler is principally used to eliminate hard deposits on the teeth via ultrasonic energy, which has a frequency $>20 \mathrm{KHz}(6-$ 8). The electronic apex locator is an instrument that employs low alternating current in order to measure the length of root canals precisely (9-12). The electric pulp tester uses high-frequency, low-intensity electric currents to produce small discharges that evaluate the vitality of sensory fibers in the pulp tissue (13-15). The electrosurge is a unipolar or bipolar surgical instrument with cutting and cauterizing functions that employs alternate electrical currents. Within the field of dentistry, the most commonly used electrosurge instrument is unipolar with a low-intensity current and a frequency of $100 \mathrm{KHz}(3,16,17)$. The osseointegration monitoring tools Osstell ISQ and Periotest $\mathrm{M}$ are based on differing principles. The mechanism of Osstell ISQ relies on the resonance frequency analysis of a wave applied to the implant surface $(5-15 \mathrm{KHz}$ and $1 \mathrm{~V}$ peak ampli- 
tude) (18-20). Periotest $\mathrm{M}$ carries out measurements via an electromagnetic mechanism that requires high-frequency energy for its internal functioning; however, the external emissions are very low (19-21).

Owing to their wide clinical applications and the levels of scientific evidence associated with their therapeutic results, the use of CIEDs has spread considerably. In the USA, the number of implanted CIEDs rose from 9,000 in 1990 to 143,000 in 2005 (22). Similar increases have been observed in European countries (23). The surge of patients with CIEDs has made it necessary to establish a consensus concerning their compatibility with certain electronic instruments employed in the field of clinical dentistry. At present, there is some controversy in the literature with respect to the possible interference of these electronic instruments with PMs and/or ICDs.

The aim of the present in vitro study was to examine the behavior of CIEDs under the influence of electronic and electromagnetic equipment employed in the field of dentistry.

\section{Material and methods}

This in vitro study was jointly designed by the Odontostomatology Department at the University of Barcelona and the Arrhythmia Unit of the Cardiology Service at the Bellvitge University Hospital (University of Barcelona).

With respect to the inclusion criteria, all electronic dental instruments tested in the study were required to possess the capacity to generate electrical or electromagnetic fields derived from their mechanisms of action. In addition, manufacturers had counterindicated their use in patients with CIEDs.

The following equipment was employed: an electrosurge (XO Odontosurge; XO Care, Hørsholm, Denmark); an electric pulp tester (Denlux B 1000 Pulppen; Dental Electronic, Ballerup, Denmark); an ultrasonic piezoelectric dental scaler (Satelec Suprasson P5 Booster; Acteon Group, Merignac Cedex, France); an electronic apex locator (Morita Root ZX; Morita, Irvine, CA, USA); and the osseointegration monitoring tools, Periotest M (Medizintechnik Gulden, Modautal, Germany) and Osstell ISQ (Osstell AB, Göteborg, Sweden).

All the CIEDs fulfilled the following inclusion criteria: the generator was new, and, during a previous function test, presented no errors or structural defects.

Three different types and manufacturers of PM [Medtronic Adapta DR ADDR01 (Medtronic, Minneapolis, MN, USA), Boston Scientific Insignia I Ultra (Boston Scientific, Natick, MA, USA), and Biotronik Estella SR-T (Biotronik SE, Berlin, Germany)], and three different types and manufacturers of ICD [Medtronic Secura DR (Medtronic), Boston Scientific Teligen 100 (Boston Scientific), and Biotronik Lumax 540 VR-T DX (Biotronik SE), were included in the study (Fig. 1). The new electrode leads with normal insulation were Capsure Fix MRI 5086 (Medtronic) for the PMs and Sprint 6932 (Medtronic) for the ICDs. The electrode leads with deteriorated insulation were 5038 VDD (Medtronic) for the PMs and Sprint Fidelis 6949 (Medtronic) for the ICDs. Bipolar electrode leads were employed in all the experiments.

The study was performed with a simulated model made of Forex (Airex AG, Sins, Switzerland), a plastic derived from expanded polyvinyl chloride (PVC). The model reproduced a number of life-size anatomic structures of reference, such as the thorax, neck, and lower jaw. It was filled with a solution of $0.4 \%$ saline in order to obtain an electrical impedance similar to that in the human body. The CIEDs were placed with their electrode leads in positions corresponding to where these leads would be placed in vivo (Fig. 1A,B).

The following variables were taken into consideration: application distance $(d A)$ and application time $(t A)$ of the instruments; dental equipment type; the type and manufacturer of the CIEDs; and the state of the insulation of the electrode leads of the CIEDs: normal $(n I)$ vs. deteriorated $(d I)$. Insulation abrasion has been reported as a common problem affecting the leads of silicone cardiac implantable devices. Abrasions may occur when a lead is in contact with the pulse generator in the pocket (can abrasion), other leads (lead-to-lead abrasion), other devices (annuloplasty ring), and anatomic structures (clavicle, rib). The implantation procedure through the jugular veins increases the risk of electrode lead bends along the way to the generator and may also eventually induce breaks in its insulation $(24,25)$.

The dental equipment was set at pulse mode - on/off in the tests with the variable $d A$ in order to test the most critical phases of the CIEDs that occur when these devices are switched on and off. In the tests with the variable $t A$, the instruments were continuously set at the 'on' mode. In all testing the dental equipment was set at maximum potency and the CIEDs were programmed to maximum sensitivity mode.

A positive control - direct contact of an electrosurge with a CIED - which always induced electromagnetic interference, was established. The negative control corresponded to the normal functioning of the CIEDs, as reflected in their corresponding electrocardiography register.

The experiments with the variable $d A$ were performed with electrode lead insulation in normal $(d A n I)$ and deteriorated $(d A d I)$ conditions. The tests for $d A n I$ were carried out at $20 \mathrm{~cm}$ from the $\mathrm{PM} / \mathrm{ICD}$, at $1 \mathrm{~cm}$ from the $\mathrm{PM} /$ ICD, and at $1 \mathrm{~cm}$ from the electrode tip (1 cm ET). The tests for the $d A d I$ were the same as for the $d A n I$ with an additional test at $1 \mathrm{~cm}$ from the deteriorated insulation region $(1 \mathrm{~cm} \mathrm{Fx})$. The experiments with the variable $t A$ were also performed with the electrode lead insulation in normal $(t A n I)$ and deteriorated $(t A d I)$ conditions. In both cases there was continuous application of the instrument for $10 \mathrm{~s}$ at $20 \mathrm{~cm}$ from the PM/ICD. In the tests where electromagnetic interference was observed, the time period of application was increased to $60 \mathrm{~s}$. All tests were carried out three times for each of the dental instruments studied, in agreement with the principles of Repeatability for the Validation for Analytical Procedures (26), providing a final total of 972 tests/observations.

All tests were monitored with specific telemetry connections for each CIED: Carelink Programmer - Model 2090 (Medtronic), Zoom Latitude 3120 (Boston Scientific), and Renamic (Biotronik SE). The electrocardiography results were printed and evaluated with the assistance of an electrophysiologist (Fig. 1C). Data from each test were registered as binary, according to whether or not interference was produced, the class of electromagnetic interference, and its category (degree of severity). For the PMs/ICDs, the electromagnetic interference categories were: electrical noise, electrical reset, deprogramming, and short- and 


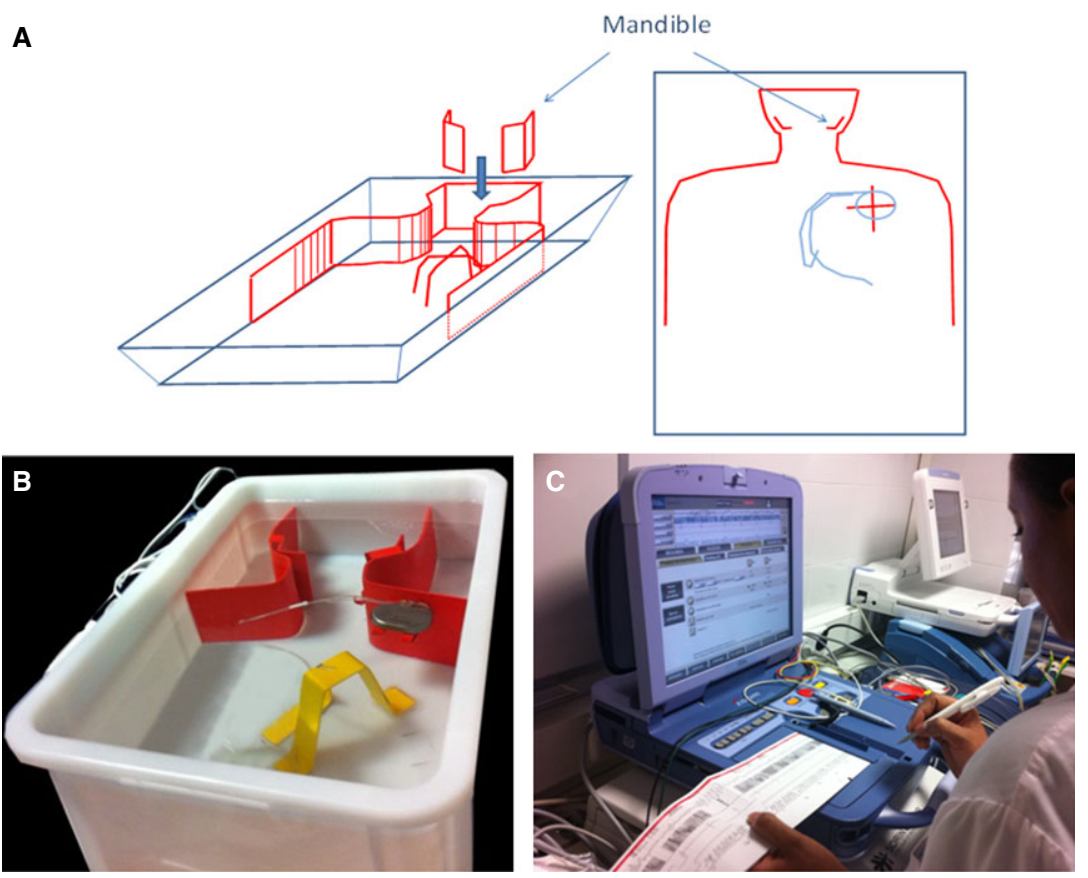

Fig. 1. (A) Diagram showing the simulated model in which the implants with cardiac activity were positioned for the in vitro experiments. (B) Simulated model with the cardiac implantable electrical device (CIED) and its corresponding electrode lead positioned to reproduce the situation in vivo. (C) Programmers with telemetry connections used to monitor the behavior of each CIED model during the study.

long-lasting stimulation inhibition. Inappropriate discharge was considered as electromagnetic interference exclusively for the ICDs and was a consequence of a false signal incorrectly interpreted as an arrhythmia. Classification of the severity of the observed interference was determined by an electrophysiologist with respect to possible clinical repercussions: absence, no interference; light, electrical noise or reset; moderate, deprogramming; severe, shortlasting stimulation inhibition ( $\leq 3$ pacings); and very severe, inappropriate discharge and long-lasting stimulation inhibition ( $>3$ pacings).

Statistical analysis was performed using the chi-square test or Fisher's exact test, as appropriate. The level of statistical significance for all the tests was $P<0.05$. In the case of double interference, only the most severe one was taken into consideration, in order to avoid errors and redundant data.

\section{Results}

\section{Dental equipment variable}

During analysis of the dental instruments, all, at some time, showed the capacity to induce electromagnetic interference in the CIEDs. With respect to the severity of the interference, significant differences were observed among the different instruments tested $(P<0.001)$. In the light and moderate categories the greatest amount of electromagnetic interference was triggered by the electrosurge. In the severe category, however, it was the electric pulp tester that caused the most electromagnetic interference (Table 1).

\section{Distance of application variable $(d A)$}

With respect to the $d A$, the quantity of interference induced in the CIEDs was statistically significant for all the dental equipment $(P<0.001)$.

For the ICDs, the electric pulp tester and ultrasonic piezoelectric dental scaler displayed significant differences in the amount of electromagnetic interference induced according to the distance of application $(P<0.001)$ and $(P=0.002)$, respectively. The electronic apex locator, electrosurge, Osstell ISQ, and Periotest $\mathrm{M}$ did not, however, present significant differences for this variable $(P>0.05)$. The greatest amount of electromagnetic interference was produced $1 \mathrm{~cm}$ from the area where the electrode lead insulation had deteriorated $(1 \mathrm{~cm} \mathrm{Fx})(P<0.001)$ (Table 2).

In the case of PMs, the electric pulp tester $(P<0.001)$, Osstell ISQ $(P=0.001)$, Periotest M $(P=0.003)$, and ultrasonic piezoelectric dental scaler $(P=0.005)$ displayed significant differences in the amount of electromagnetic interference induced, according to the distance of application. However, the electronic apex locator and electrosurge did not $(P>0.05)$. A significantly greater quantity of electromagnetic interference was associated with a distance of $1 \mathrm{~cm}$ from the electrode tip $(1 \mathrm{~cm} \mathrm{ET})(P<0.001)$ (Table 2).

The distance between the CIED, located in the infraclavicular region, and the oral cavity is generally about $20 \mathrm{~cm}$. At this distance only two electric noises (electromagnetic interference light category), which 
Table 1

Electromagnetic interference category of severity adjusted according to the dental equipment tested

\begin{tabular}{|c|c|c|c|c|c|c|c|c|c|c|c|c|c|c|c|}
\hline \multirow[b]{3}{*}{ Dental equipment } & \multicolumn{12}{|c|}{ Degree of severity } & & & \\
\hline & \multicolumn{3}{|c|}{ Light } & \multicolumn{3}{|c|}{ Moderate } & \multicolumn{3}{|c|}{ Severe } & \multicolumn{3}{|c|}{ Very severe } & \multicolumn{3}{|c|}{ Total } \\
\hline & $\bar{n}$ & $\% \dagger$ & $P^{*}$ & $\bar{n}$ & $\%^{\dagger}$ & $P^{*}$ & $\bar{n}$ & $\%^{\dagger}$ & $P^{*}$ & $\bar{n}$ & $\%{ }^{\dagger}$ & $P^{*}$ & $n$ & $\%^{\dagger}$ & $P^{*}$ \\
\hline EPT & 18 & 12.4 & & 0 & 0.0 & & 33 & 49.25 & & 0 & 0.0 & & 51 & 23.7 & \\
\hline EAL & 15 & 10.3 & & 0 & 0.0 & & 0 & 0.0 & & 0 & 0.0 & & 15 & 6.97 & \\
\hline OT & 33 & 22.8 & & 0 & 0.0 & & 6 & 8.96 & & 0 & 0.0 & & 39 & 18.14 & \\
\hline ES & 37 & 25.5 & $<0.001$ & 3 & 100.0 & $<0.001$ & 16 & 23.88 & $<0.001$ & 0 & 0.0 & $<0.001$ & 56 & 26.04 & $<0.001$ \\
\hline PT & 18 & 12.4 & & 0 & 0.0 & & 6 & 8.96 & & 0 & 0.0 & & 24 & 11.2 & \\
\hline UDS & 24 & 16.6 & & 0 & 0.0 & & 6 & 8.96 & & 0 & 0.0 & & 30 & 13.95 & \\
\hline Total & 145 & 100.0 & & 3 & 100.0 & & 67 & 100.0 & & 0 & 0.0 & & 215 & 100.0 & \\
\hline
\end{tabular}

EAL, electronic apex locator; EPT, electric pulp tester; ES, electrosurge; OT, Osstell ISQ; PT, Periotest M; UDS, ultrasonic dental scaler. * $P$ value (Fisher's exact test).

$\dagger \%$ total interference according to degree of severity

were induced by electrosurge, were reported in PMs. For the ICDs, 24 electric noises were observed (electromagnetic interference light category), which were induced by various dental instruments (Table 2).

\section{Time of application variable $(t A)$}

In the analysis of $t A$, it was observed that lengthening the time from $10 \mathrm{~s}$ to $60 \mathrm{~s}$ did not modify the amount of electromagnetic interference for any of the CIEDs $(P=1.000)$, a result that was reported for both normal $(P=1.000)$ and deteriorated $(P=1.000)$ electrode lead insulation.

\section{Type of CIED variable (PM vs. ICD)}

In the analysis of the type of CIED variable with respect to interference and its degree of severity, overall the ICDs presented the greatest amount $(P<0.001)$ and the largest number of electromagnetic interference in the category light $(P<0.001)$. The PMs, however, displayed the greatest amount of moderate and severe interference $(P<0.001)$ (Table 3).

\section{Manufacturer of CIED variable}

In the analysis of the different manufacturers of CIEDs, significant differences were observed for electromagnetic interference $(P<0.001)$. In the PMs the number of interferences were 75 for Boston Scientic, 38 for Biotronik, and 24 for Medtronic, and, in the ICDs, were 114 for Boston Scientic, 18 for Medtronic, and 15 for Biotronik $(P<0.001)$ (Table 4$)$.

\section{Electrode lead insulation integrity ( $n /$ vs. $d$ ) variable}

In the analysis of the electrode lead insulation integrity variation (normal vs. deteriorated) statistical significance was globally observed in the number of interferences $\quad(P<0.001)$, with higher electromagnetic interference values when the insulation was deteriorated. Significant differences were also reported when the variable $n I$ vs. $d I$ was evaluated with respect to $d A$ $(P<0.001)$ and $t A(P=0.032)$, again with higher electromagnetic interference values for deteriorated insulation.

\section{Discussion}

Pacemakers and ICDs are sensitive to external electromagnetic interference. In spite of the fact that the smaller, present-day CIEDs have better protective characteristics, their normal function can be affected by some electronic dental instruments that emit electromagnetic waves (5).

To the best of our knowledge, no previous studies have evaluated the electromagnetic interferences induced by either the Osstell ISQ or the Periotest M. Most dental research has been focused on electronic apex locators, ultrasonic dental scalers, electrosurge instruments, and electric pulp testers $(8,11,12,27-36)$. In reference to the electric pulp tester, the first in vivo study, performed by Woolley et al. (27), reported that this instrument provoked electromagnetic interference in PMs. Some authors observed that electric pulp testers did not induce electromagnetic interference in PMs $(28,30)$. More recently, WiLson et al. (34) stated that electric pulp testers did not induce any kind of interference in vivo in the ICDs and PMs studied. In our work, the electric pulp tester was the instrument that triggered the greatest number of interferences in PMs and ICDs, and was also the device that caused the most severe electromagnetic interferences. After analyzing the discrepancies in these published studies we are of the opinion that they could be because an in vivo model was used in contrast to an in vitro one. It can be difficult with in vivo studies to carry out certain specific situations that are easily reproducible in an in vitro experimental model. Therefore, the model chosen for our in vitro study could explain the increased electromagnetic interference in the CIEDs.

The capacity of ultrasonic dental scalers to interfere with CIEDs has been widely studied by several authors. 
ZAPPA et al. (30) did not report in vivo interference from an ultrasonic dental scaler on PMs. PAtel et al. (33) concluded that an ultrasonic dental scaler could be employed with the patients fitted with a PM included in their study. In agreement with this finding, our research showed that, at a clinical distance of application $(20 \mathrm{~cm})$, no electromagnetic interference was recorded for the ultrasonic dental scaler with the tested PMs. In contrast, Miller et al. (31) reported in vitro the presence of electromagnetic interference with the use of an ultrasonic dental scaler in uni- and bipolar PM groups. AdAms et al. (29) specified that they had detected interference only when the ultrasonic dental scaler handpiece was at a distance of $6 \mathrm{~cm}$ or less from the PM electrode leads. We also observed electromagnetic interference when the ultrasonic dental scaler application distance was up to $1 \mathrm{~cm}$ from the PM, and up to $1 \mathrm{~cm}$ from the area where the electrode lead insulation had deteriorated.

In contrast, electromagnetic slight interferences were reported for ICDs, even at clinical application distances $(20 \mathrm{~cm})$. Roedig et al. (36) evaluated in vitro the effects of various dental instruments on PMs and ICDs and observed that ultrasonic dental scaler induced interference in all the CIEDs studied. They also reported that interference generally occurred close to the generator, especially when near the electrode leads. In our in vitro study no differences between applications at $1 \mathrm{~cm}$ from the generator vs. $1 \mathrm{~cm}$ from the electrode tip were observed. Recent in vivo research, conducted by MaIorana et al. (8), led to the conclusion that ultrasonic dental scaler did not interfere with the ICDs tested. In our study, this equipment triggered interference in the PMs and ICDs, particularly when applied near the deteriorated areas of the electrode lead insulation or the electrode tip. In 2000, the American Academy of Periodontology established clinical guidelines for ultrasonic dental scalers, recommending that they should not be employed in patients with PMs (37). However, these criteria were revoked in 2007 and, at present, there are no guidelines concerning their use in patients with a PM. Scientific evidence suggests that normal clinical use of this dental ultrasonic equipment has no effect on CIEDs $(8,29$, $32,33,35,38)$. In our study, the ultrasonic dental scalers, at a clinical distance of application $(20 \mathrm{~cm})$, only produced light electromagnetic interference in the ICDs.

The electrosurge has been tested in several studies. Woolley et al. (27) reported that electrosurge provoked electromagnetic interference in PMs in vivo. In contrast, BRAND et al. (35), in an in vitro study with electromagnetic equipment, including the electrosurge, concluded that the instruments tested did not emit any electromagnetic interferences of risk for the CIEDs analyzed. Other authors confirm these results (30). It should be recognized that, in our study, the electrosurge triggered electromagnetic interference in both types of CIEDs, particularly in the PMs, and above all when applied at $1 \mathrm{~cm}$ from the generator or $1 \mathrm{~cm}$ from the electrode tip. Indeed, the manufac- 
Table 3

Electromagnetic interference severity categories adjusted according to the type of cardiac implantable electrical device (CIED) analyzed

\begin{tabular}{|c|c|c|c|c|c|c|c|c|c|c|c|c|c|c|c|}
\hline \multirow[b]{3}{*}{ CIED } & \multicolumn{12}{|c|}{ Degree of severity } & & & \\
\hline & \multicolumn{3}{|c|}{ Light } & \multicolumn{3}{|c|}{ Moderate } & \multicolumn{3}{|c|}{ Severe } & \multicolumn{3}{|c|}{ Very severe } & \multicolumn{3}{|c|}{ Total } \\
\hline & $n$ & $\%^{\dagger}$ & $P^{*}$ & $n$ & $\%^{\dagger}$ & $P^{*}$ & $n$ & $\%^{\dagger}$ & $P^{*}$ & $n$ & $\%^{\dagger}$ & $P^{*}$ & $n$ & $\%^{\dagger}$ & $P^{*}$ \\
\hline PM & 28 & 19.3 & & 3 & 100.0 & & 52 & 77.6 & & 0 & 0.0 & & 83 & 38.6 & \\
\hline ICD & 117 & 80.7 & $<0.001$ & 0 & 0.0 & $<0.001$ & 15 & 22.4 & $<0.001$ & 0 & 0.0 & $<0.001$ & 132 & 61.4 & $<0.001$ \\
\hline Total & 145 & 100.0 & & 3 & 100.0 & & 67 & 100.0 & & 0 & 0.0 & & 215 & 100.0 & \\
\hline
\end{tabular}

CIED, cardiac implantable electrical device; ICD, implantable cardioverter defibrillator; PM, pacemaker.

* $P$ value (Fisher's exact test).

$\dagger \%$ total of interference according to degree of severity.

turer, Boston Scientific, does warn that certain precautions must be taken during the use of an electrosurge in order to minimize the risk of interference (39).

There are various publications which make reference to the possible effects of the electronic apex locator on PMs and ICDs. Garofalo et al. (32), in their in vitro study, concluded that electronic apex locator could be used without risk in patients with PMs. PATEl et al. (33) concluded that electronic apex locator could be employed with patients fitted with a PM who were included in their study. In similar manner, Wilson et al. (34) observed in vivo that electronic apex locator did not induce any kind of interference on the PMs and ICDs studied. More recently, Gomez et al. (11) reported that electromagnetic interference only occurs in vitro when the electronic apex locators are applied close to the electrode tip, and occasionally near PMs. IDZAHI et al. (12), in their in vitro research, did not find any interference from the electronic apex locators on the ICDs studied. According to our results, the electronic apex locator is also considered to be a safe instrument when used at a clinical application distance $(20 \mathrm{~cm})$.

Publications comparing PM and ICD behavior with respect to electronic dental equipment are scarce (36). In our in vitro study, the ICDs displayed a greater quantity of light electromagnetic interference, whereas that presented by the PMs was moderate and severe. In this respect, PINSKI et al. (40) affirmed that the ICDs have greater sensitivity as they are designed for early detection of ventricular tachyarrhythmia, which would account for the higher electromagnetic interference in our findings.

Regarding classification of interference, some authors agreed to categorize the severity of each stimulation inhibition according to the PM dependence and the duration of this induced inhibition on CIEDs $(1,41)$. In our study, short-lasting stimulation inhibitions $(\leq 3$ pacings) were classified in the severe category. On the other hand, long-lasting stimulation inhibitions (>3 pacings) were classified in the very severe category.

Table 4

Electromagnetic interference adjusted according to the cardiac implantable electrical device (CIED) manufacturers analyzed

\begin{tabular}{|c|c|c|c|c|c|c|c|c|c|c|c|c|c|c|c|c|c|c|}
\hline \multirow[b]{3}{*}{ Manufacturer } & \multicolumn{15}{|c|}{ Electromagnetic interference variable } & & & \\
\hline & \multicolumn{3}{|c|}{ Elec. noise } & \multicolumn{3}{|c|}{ Elec. reset } & \multicolumn{3}{|c|}{ Deprogram. } & \multicolumn{3}{|c|}{ Sti. inhibition } & \multicolumn{3}{|c|}{ Inap. disc ${ }^{\ddagger}$} & \multicolumn{3}{|c|}{ Total } \\
\hline & $n$ & $\%^{\dagger}$ & $P^{*}$ & $n$ & $\%^{\dagger}$ & $P^{*}$ & $n$ & $\%^{\dagger}$ & $P^{*}$ & $n$ & $\%^{\dagger}$ & $P^{*}$ & $n$ & $\%^{\dagger}$ & $P^{*}$ & $n$ & $\%^{\dagger}$ & $P^{*}$ \\
\hline \multicolumn{19}{|l|}{ Global } \\
\hline Biotronik & 28 & 9.9 & $<0.001$ & 0 & 0.0 & nc & 3 & 11 & 0.110 & 22 & 7.7 & 0.401 & 0 & 0.0 & $\mathrm{nc}$ & 53 & 18.7 & $<0.001$ \\
\hline Boston & 162 & 57.0 & & 0 & 0.0 & & 0 & 0.0 & & 27 & 9.5 & & 0 & 0.0 & & 189 & 66.5 & \\
\hline Medtronic & 24 & 8.5 & & 0 & 0.0 & & 0 & 0.0 & & 18 & 6.3 & & 0 & 0.0 & & 42 & 14.8 & \\
\hline \multicolumn{19}{|l|}{ PM } \\
\hline Biotronik & 19 & 6.7 & $<0.001$ & 0 & 0 & $\mathrm{nc}$ & 3 & 1.1 & 0.110 & 16 & 5.6 & $<0.005$ & - & - & - & 38 & 13.4 & $<0.001$ \\
\hline Boston & 48 & 16.9 & & 0 & 0 & & 0 & 0 & & 27 & 9.5 & & - & - & & 75 & 26.4 & \\
\hline Medtronic & 15 & 5.3 & & 0 & 0 & & 0 & 0 & & 9 & 3.2 & & - & - & & 24 & 8.5 & \\
\hline \multicolumn{19}{|l|}{ ICD } \\
\hline Biotronik & 9 & 3.2 & $<0.001$ & 0 & 0 & $\mathrm{nc}$ & 0 & 0 & $\mathrm{nc}$ & 6 & 2.1 & $<0.004$ & 0 & 0 & $\mathrm{nc}$ & 15 & 5.3 & $<0.001$ \\
\hline Boston & 114 & 40.1 & & 0 & 0 & & 0 & 0 & & 0 & 0 & & 0 & 0 & & 114 & 40.1 & \\
\hline Medtronic & 9 & 3.2 & & 0 & 0 & & 0 & 0 & & 9 & 3.2 & & 0 & 0 & & 18 & 6.4 & \\
\hline
\end{tabular}

Deprogram., deprogramming; Elec., electrical; EMI, electromagnetic interference; ICD, implantable cardioverter defibrillator; Inap. disc, inappropriate discharge; nc, not calculated; PM, pacemaker; Sti. inhibition, stimuation inhibition.

* $P$ value (Fisher's exact test).

$\dagger \%$ according to total EMI $(n=284)$.

*EMI inappropriate discharge was found only in the ICDs. 
It has been reported, by some researchers, that insulation abrasion is a common problem affecting the silicone electrode leads of CIEDs $(24,25)$. According to our results, the state of insulation of the electrode lead was a variable that had a considerable effect on the amount of electromagnetic interference registered; deterioration increased the quantity of electromagnetic interference.

For in vitro experimental protocols, a bucket filled with a saline solution that has had its impedance adjusted to human body values is generally used (11, $12,31,35,36)$. In our study, however, in order to achieve conditions close to those found in vivo, a simulated model was designed. Moreover, material that reproduced some life-size anatomic structures of reference (thorax, heart, neck, and mandible) was employed, which permitted the correct positioning of the CIEDs and their electrode leads.

With reference to the effective in vivo reproducibility of in vitro results, some authors have stated that, first and foremost, electric and magnetic fields decrease inversely with the square of the distance from the source (2). Moreover, the surrounding body tissues further shield the device from electromagnetic interference (38). In our study we reproduced human body features; nevertheless, in vivo tissues, such as skin, fat, muscle, bone, and teeth, may additionally hinder the conduction of electromagnetic currents. It is therefore probable that under in vivo conditions there is less electromagnetic interference induced by electric pulp tester, electronic apex locator, ultrasonic dental scaler, unipolar electrosurge, Osstell ISQ and Periotest M, particularly in heavily built patients or in those with a high body mass.

RoEDIG et al. (36) acknowledged that a limitation of their research was to have studied only one PM/ICD manufacturer. DoDinot et al. (42) observed differences between PMs produced by different manufacturers and concluded that the PM produced by Medtronic was the most resistant with respect to electromagnetic interference. In our study, differences in electromagnetic interference were observed in the CIEDs according to their manufacturer; those with the lowest electromagnetic interference were the PMs and ICDs produced by Medtronic and Biotronik, respectively.

In summary, our in vitro model permitted a precise reproduction of in vivo conditions for the experiments performed. The possibility of carrying out extreme tests near the CIEDs and the electrode tips demonstrated that under certain conditions some dental equipment can trigger electromagnetic interference in PMs and/or ICDs. Our results show that at a clinical application distance $(20 \mathrm{~cm})$, the electronic dental equipment tested only provoked light interference (electric noise) in the CIED examined, irrespective of manufacturer. Therefore, we can conclude that the dental instruments analyzed in our study may be used in clinical dentistry for patients with PMs and ICDs. This study could be of help in redefining standards and guidelines concerning the use of electronic dental equipment in patients with cardiac implanted electrical devices.
Acknowledgements - The authors thank Glòria Pérez, MD, PhD, and Marco Cornejo, DDS, PhD, for their help with the statistical analyses. The authors express their gratitude to Juan Benito Tur, Albert Gómez, Omar Ferrús and Ramón Casanovas for their excellent technical assistance. We also appreciate the support provided by Medtronic, Biotronik SE KG, Osstell AB and the staff of the Arrhythmias Unit at Bellvitge University Hospital (University of Barcelona).

Conflicts of interest - The authors declare that they have no conflict of interest.

\section{References}

1. Misiri J, Kusumoto F, Goldschlager N. Electromagnetic interference and implanted cardiac devices: the nonmedical environment (part I). Clin Cardiol 2012; 35: 276-280.

2. Pinski SL, Trohman RG. Interference in implanted cardiac devices, part I. Pacing Clin Electrophysiol 2002; 25: 1367-1381.

3. Pinski SL, Trohman RG. Interference in implanted cardiac devices, part II. Pacing Clin Electrophysiol 2002; 25: 1496 1509.

4. Rozner MA. Review of electrical interference in implanted cardiac devices. Pacing Clin Electrophysiol 2003; 26: 923-925.

5. Boston Scientific Co. Dental equipment and implantable pacemakers and defibrillators. Natick, MA: Boston Scientific Co, 2009.

6. Breininger DR, O'Leary TJ, Blumenshine RV. Comparative effectiveness of ultrasonic and hand scaling for the removal of subgingival plaque and calculus. J Periodontol 1987; 58: 9-18.

7. Satelec Acteon Group. Satelec P5 booster. Operation manual. Merignac, Cedex: Satelec Acteon Group, 2004.

8. Maiorana C, Grossi GB, Garramone RA, Manfredini R, SANToro F. Does ultrasonic dental scaler interfere with implantable cardioverter defibrillators? An in vivo testing $J$ Dent 2013; 41: 955-959.

9. D'assunção Fl, De Alburquerque DS, Salazar-Silva JR, De Queiroz Ferreira LC, Bezerra PM. The accuracy of root canal measurements using the Mini Apex Locator and Root ZX-II: an evaluation in vitro. Oral Surg Oral Med Oral Pathol Oral Radiol Endod 2007; 104: 50-53.

10. Morita J. Root $Z X$. Operation instructions. Irvine, CA: J. Morita MFG Corp, 2009.

11. Gomez G, Duran-Sindreu F, Jara Clemente F, Garofalo RR, Garcia M, Bueno R, Roig M. The effects of six electronic apex locators on pacemaker function: an in vitro study. Int Endod J 2013; 46: 399-405.

12. Idzahi K, De Cock CC, Shemesh H, Brand HS. Interference of electronic apex locators with implantable cardioverter defibrillators. J Endod 2014; 40: 277-280.

13. Dental Electronic. Denlux B 1000 Pulppen. User's manual. Ballerup: Dental Electronic, 2007.

14. Lin J, Chandler NP. Electric pulp testing: a review. Int Endod $J$ 2008; 41: 365-374.

15. Jafarzadeh H, Аввоtт PV. Review of pulp sensibility tests. Part II: electric pulp tests and test cavities. Int Endod J 2010; 43: 945-958.

16. XO CARE. Odontosurge. Operation manual. Hørsholm: XO Care, 2002.

17. Dawes JC, Mahabir RC, Hillier K, Cassidy M, De Haas W, Gillis AM. Electrosurgery in patients with pacemakers/ implanted cardioverter defibrillators. Ann Plast Surg 2006; 57: 33-36.

18. Meredith N, Cawley P, Alleyne D. The application of modal vibration analysis to study bone healing in vivo. J Dent Res 1994; 73: 793.

19. Lachmann S, Jäger B, Axmann D, Gomez-Roman G, GroTEN M, WeBER H. Resonance frequency analysis and damping capacity assessment. Part 1: an in vitro study on measurement reliability and a method of comparison in the determination 
of primary dental implant stability. Clin Oral Implan Res 2006; 17: 75-79.

20. Osstell AB. Osstell ISQ. User's guide. Göteborg: Osstell AB, 2011.

21. Medizintechnik Gulden. Periotest M. User's manual. Modautal: Medizintechnik Gulden, 2007.

22. Greenspon aJ, Patel JD, Lau E, Ochoa Ja, Frisch DR, Ho RT, Pavri BB, Kurtz SM. Trends in permanent pacemaker implantation in the United States from 1993 to 2009: increasing complexity of patients and procedures. $J$ Am Coll Cardiol 2012; 60: 1540-1545.

23. European Heart Network and European Society of Cardiology. European cardiovascular disease statistics. Sophia-Antipolis, Cedex: European Heart Network and European Society of Cardiology, 2012. http://www.escardio.org/about/documents/eu-cardiovascular-disease-statistics-2012.pdf (May 2014).

24. Hauser RG, Abdelhadi RH, Mcgriff DM, Kallinen Retel L. Failure of a novel-silicone polyurethane copolymer (Optim) to prevent implantable cardioverter-defibtillator lead insulation abrasions. Europace 2013; 15: 278-283.

25. Gradaus R, Breithardt G, Bocker D. ICD leads: design and chronic dysfunctions. Pacing Clin Electrophysiol 2003; 26: 649-657.

26. European Medicines Agency. Validation for analytical procedures: text and methodology step-5. London: European Medicines Agency, 1995. http//:www.ema.europa.eu (May 2014).

27. Woolley LH, Woodworth J, Dobbs JL. A preliminary evaluation of the effects of electrical pulp testers on dogs with artificial pacemakers. $J$ Am Dent Assoc 1974; 89: 10991101.

28. Simon AB, Linde B, Bonnette GH, Schlentz RJ. The individual with a pacemaker in the dental environment. $J \mathrm{Am}$ Dent Assoc 1975; 91: 1224-1229.

29. Adams D, Fulfor N, Beechy J, Maccarthy J, Stephens M. The cardiac pacemaker and ultrasonic scalers. $\mathrm{Br}$ Dent $J$ 1982; 152: 171-173.

30. Zappa U, Studer M, Merkle A, Graf H, Simona C. Effect of electrically powered dental devices on cardiac parameter function in humans. Parodontologie 1991; 2: 299-308.
31. Miller CS, Leonelli FM, Latham E. Selective interference with pacemaker activity by electrical dental devices. Oral Surg Oral Med Oral Pathol Oral Radiol Endod 1998; 85: 33-36.

32. Garofalo RR, Ede EN, Dorn SO, Kuttler S. Effect of electronic apex locators on cardiac pacemaker function. $J$ Endod 2002; 28: 831-833.

33. Patel D, Glick M, Lessard E. Absence of in vivo effects of dental instruments on pacemaker function. Oral Surg Oral Med Oral Pathol Oral Radiol Endod 2005; 99: 430.

34. Wilson BL, Broberg C, Baumgartner JC, Harris C, Kron J. Safety of electronic apex locators and pulp testers in patients with implanted cardiac pacemakers or cardioverter/ defibrillators. $J$ Endod 2006; 32: 847-852.

35. Brand HS, Entjes ml, Nieuw Amerogen AV, Van Der Hoeff EV, Schrama TA. Interference of electrical dental equipment with implantable cardioverter-defibrillators. $\mathrm{Br}$ Dent $J$ 2007; 203: 577-579.

36. Roedig JJ, Shah J, Elayi CS, Miller CS. Interference of cardiac pacemaker and implantable cardioverter-defibrillator activity during electronic dental device use. J Am Dent Assoc 2010; 141: 521-526.

37. Drisko Cl, Cochran Dl, Blieden T, Bouwsma OJ, Cohen RE, Damoulis P, Fine JB, Greenstein G, Hinrichs J, Somerman MJ, Iacono V, Genco RJ, Research, Science and Therapy Committee of the American Accademy of Periodontology. Position paper: sonic and ultrasonic scalers in periodontics. Research, Science and Therapy Committee of the American Academy of Periodontology. J Periodontol 2000; 71: 792-801.

38. Glikson M, Hayes DL. Cardiac pacing. A review. Med Clin North Am 2001; 85: 369-421.

39. Boston Scientific Co. Electrocautery and implantable device systems. Natick, MA: Boston Scientific Co, 2008.

40. Pinski SL, Trohman RG. Permanent pacing via implantable defibrillators. Pacing Clin Electrophysiol 2000; 23: 1667-1681.

41. Misiri J, Kusumoto F, Goldschlager N. Electromagnetic interference and implanted cardiac devices: the medical environment (part II). Clin Cardiol 2012; 35: 321-328.

42. Dodinot B, Godenir J-P, Costa AB. Electronic article surveillance: a possible danger for pacemaker patients. Pace 1993; 16: 46-53. 